https://doi.org/10.15407/ujpe64.11.991

S. MIGNEMI ${ }^{1,2}$

${ }^{1}$ Dipartimento di Matematica e Informatica, Universitá di Cagliari, (viale Merello 92,09123 Cagliari, Italy; e-mail: smignemi@unica.it)

2 INFN, Sezione di Cagliari

(Cittadella Universitaria, Monserrato, Italy)

\title{
THE SNYDER MODEL AND QUANTUM FIELD THEORY ${ }^{1}$
}

We review the main features of the relativistic Snyder model and its generalizations. We discuss the quantum field theory on this background using the standard formalism of noncommutative QFT and discuss the possibility of obtaining a finite theory.

Ke ywords: Snyder model, noncommutative field theory.

\section{Introduction}

Since the origin of quantum field theory (QFT), the proposals have been advanced to add a new scale of length to the theory in order to solve the problems connected to UV divergences. Later, the attempts to build a theory of quantum gravity have proved the necessity of introducing a length scale, which has been identified with the Planck length $L_{p}=\sqrt{\frac{\hbar G}{c^{3}}} \sim 1.6 \times 10^{-35} \mathrm{~m}[1,2]$. A naive application of this idea, like a lattice field theory, would however break the Lorentz invariance. A way to reconcile the discreteness of spacetime with the Lorentz invariance was proposed by Snyder [3,4] long time ago. This was the first example of a noncommutative geometry: the length scale should enter the theory through the commutators of spacetime coordinates.

Noncommutative geometries were, however, not investigated for a long time, but they revived due to mathematical $[5,6]$ and physical $[7,8]$ progresses. Their present understanding is based on the formalism of Hopf algebras [9]. In particular, QFT on noncommutative backgrounds has been largely studied $[7,8]$. In most cases, a surprising phenomenon, called UV/IR mixing, occurs: the counterterms needed for the UV regularization diverge for vanishing incoming momenta, inducing an IR divergence.

Noncommutative geometries also admit a sort of dual representation on the momentum space in theories of doubly special relativity (DSR) [10]. Here, a fundamental mass scale is introduced, that causes the curvature of the momentum space $[12,13]$, and

(C) S. MIGNEMI, 2019

ISSN 2071-0194. Ukr. J. Phys. 2019. Vol. 64, No. 11 the deformation of both the Poincaré group and the dispersion relations of the particles. The Snyder model can also be seen as a DSR model, where the Poincaré invariance and the dispersion relations are undeformed.

As mentioned above, Snyder's idea was almost abandoned with the introduction of renormalization techniques, with the exception of some Russian authors in the sixties [14-19]. It revived recently, when the noncommutative geometry became an important topic of research. However, in spite of several attempts using various methods [14-21], the issue of finiteness of the Snyder field theory has not been established up to now. Here, we review an attempt to investigate this topic using the formalism of noncommutative QFT [22-24].

\section{The Snyder Model}

The most notable feature of the Snyder model is that, in contrast with most examples of the noncommutative geometry, it preserves the full Poincaré invariance. In fact, it is based on the Snyder algebra, a deformation of the Lorentz algebra acting on the phase space, generated by positions $x_{\mu}$, momenta $p_{\mu}$, and Lorentz generators $J_{\mu \nu}$, that obey the Poincaré commutation relations

$$
\begin{aligned}
& {\left[J_{\mu \nu}, J_{\rho \sigma}\right]=i\left(\eta_{\mu \rho} J_{\nu \sigma}-\eta_{\mu \sigma} J_{\nu \rho}+\eta_{\nu \rho} J_{\mu \sigma}-\eta_{\nu \sigma} J_{\mu \rho}\right)} \\
& {\left[p_{\mu}, p_{\nu}\right]=0, \quad\left[J_{\mu \nu}, p_{\lambda}\right]=i\left(\eta_{\mu \lambda} p_{\nu}-\eta_{\lambda \nu} p_{\mu}\right)}
\end{aligned}
$$

1 This work is based on the results presented at the XI BolyaiGauss-Lobachevskii (BGL-2019) Conference: Non-Euclidean, Noncommutative Geometry and Quantum Physics. 
together with the standard Lorentz action on the position

$\left[J_{\mu \nu}, x_{\lambda}\right]=i\left(\eta_{\mu \lambda} x_{\nu}-\eta_{\nu \lambda} x_{\mu}\right)$,

and a deformation of the Heisenberg algebra (preserving the Jacobi identities),

$\left[x_{\mu}, x_{\nu}\right]=i \beta J_{\mu \nu}, \quad\left[x_{\mu}, p_{\mu}\right]=i\left(\eta_{\mu \nu}+\beta p_{\mu} p_{\nu}\right)$,

where $\beta$ is a parameter of the order of the square of the Planck length, and $\eta_{\mu \nu}=\operatorname{diag}(-1,1,1,1)$. The generators $J_{\mu \nu}$ are realized in the standard way as $J_{\mu \nu}=x_{\mu} p_{\nu}-x_{\nu} p_{\mu}$.

In contrast with most models of the noncommutative geometry, commutators (4) are functions of the phase space variables: this allows them to be compatible with a linear action of the Lorentz symmetry on the phase space. However, translations act in a nontrivial way on position variables.

It is important to remark that, depending on the sign of the coupling constant $\beta$, two rather different models can arise:

$\beta>0$ Snyder model,

$\beta<0$ anti-Snyder model.

They have very different properties. For example, the Snyder model has a discrete spatial structure and a continuous time spectrum, while the opposite holds for the anti-Snyder one.

The subalgebra generated by $J_{\mu \nu}$ and $x_{\mu}$ is isomorphic to the de Sitter/anti-de Sitter algebra. Hence, the Snyder/anti-Snyder momentum spaces have the same geometry as the de Sitter/anti-de Sitter spacetime. In fact, the Snyder momentum space can be represented as a hyperboloid $\mathcal{H}$ of the equation

$\zeta_{A}^{2}=1 / \beta$

embedded in a 5D space of coordinates $\zeta_{A}$ with signature $(-,+,+,+,+)$ or, equivalently, as a coset space $\mathrm{SO}(1,4) / \mathrm{SO}(1,3)$.

The Snyder commutation relations are recovered through the choice of isotropic (Beltrami) coordinates on $\mathcal{H}$

$p_{\mu}=\zeta_{\mu} / \zeta_{4}$

and the identification

$x_{\mu}=M_{\mu 4}, \quad J_{\mu \nu}=M_{\mu \nu}$,

992 where $M_{A B}$ are the Lorentz generators in 5D. Note that this construction implies that $p^{2}<1 / \beta$ and, hence, the existence of a maximal mass of the order of the Planck mass for elementary particles. This is a common feature in models with a curved momentum space $[12,13]$.

The momentum space of the anti-Snyder model can be represented analogously, as a hyperboloid of Eq. (5) with $\beta<0$, embedded in a 5D space of coordinates $\zeta_{A}$ with signature $(+,-,-,-,+)$ or, equivalently, as a coset space $\mathrm{SO}(2,3) / \mathrm{SO}(1,3)$. Again, the anti-Snyder commutation relations are recovered through the choice of isotropic coordinates (6) and identification (7). An important difference from the previous case is that the momentum squared is now unbounded. In the following, we shall concentrate on the Snyder space, but most results hold also for $\beta<0$.

\section{Generalizations of the Snyder Model}

The Snyder model can be generalized by choosing different isotropic parametrizations of the momentum space, but maintaining the identification $x_{\mu}=M_{\mu 4}$. In this way, Eqs. (1)-(3) and the position commutation relations still hold, but $\left[x_{\mu}, p_{\nu}\right]$ is deformed. For example, choosing $p_{\mu}=\zeta_{\mu}$, one obtains $[25,26]$

$\left[x_{\mu}, x_{\nu}\right]=i \beta J_{\mu \nu}, \quad\left[x_{\mu}, p_{\nu}\right]=i \sqrt{1+\beta p^{2}} \eta_{\mu \nu}$.

The most general choice that preserves the Poincaré invariance is [27]

$p_{\mu}=f\left(\zeta^{2}\right) \zeta_{\mu}, \quad x_{\mu}=g\left(\zeta^{2}\right) M_{\mu 4}$.

Algebraically, the same models can also be obtained by deforming the Heisenberg algebra as [25, $26,28]$

$$
\begin{aligned}
& {\left[x_{\mu}, x_{\nu}\right]=i \beta J_{\mu \nu} \psi\left(\beta p^{2}\right),} \\
& {\left[x_{\mu}, p_{\nu}\right]=i\left[\eta_{\mu \nu} \phi_{1}\left(\beta p^{2}\right)+\beta p_{\mu} p_{\nu} \phi_{2}\left(\beta p^{2}\right)\right] .}
\end{aligned}
$$

The functions $\phi_{1}$ and $\phi_{2}$ are arbitrary, but the Jacobi identity yields

$\psi=\phi_{1} \phi_{2}-2\left(\phi_{1}+\beta p^{2} \phi_{2}\right) \frac{d \phi_{1}}{d\left(\beta p^{2}\right)}$.

A different kind of generalization is obtained by choosing a curved spacetime (de Sitter) background, imposing nontrivial commutation relations between the momentum variables,

$\left[p_{\mu}, p_{\nu}\right]=i \alpha J_{\mu \nu}$

ISSN 2071-0194. Ukr. J. Phys. 2019. Vol. 64, No. 11 
with $\alpha$ proportional to the cosmological constant. This idea goes back to Yang [29], but was later elaborated in a more compelling way in [30]. We call this generalization the Snyder-de Sitter (SdS) model.

The other commutation relations are unchanged, except for that now, by the Jacobi identities,

$$
\begin{aligned}
& {\left[x_{\mu}, p_{\nu}\right]=i\left(\eta_{\mu \nu}+\alpha x_{\mu} x_{\nu}+\beta p_{\mu} p_{\nu}+\right.} \\
& \left.+\sqrt{\alpha \beta}\left(x_{\mu} p_{\nu}+p_{\mu} x_{\nu}\right)\right) .
\end{aligned}
$$

This model depends on two invariant scales besides the speed of light, that are usually identified with the Planck mass and the cosmological constant, from which the alternative name is triply special relativity, proposed in [30]. It must be noted that, in order to have real structure constants, both $\alpha$ and $\beta$ must have the same sign. There are indications that the introduction of $\alpha$ might be necessary to obtain a well-behaved low-energy limit of quantum gravity theories [30].

An interesting property of the SdS model is its duality for the exchange $\alpha x \leftrightarrow \beta p$ [31], that realizes the Born reciprocity [32]. The phase space can be embedded in a $6 \mathrm{D}$ space as $\frac{\mathrm{SO}(1,5)}{\mathrm{SO}(1,3) \times \mathrm{O}(2)}$, if $\alpha, \beta>0$, or as $\frac{\mathrm{SO}(2,4)}{\mathrm{SO}(1,3) \times \mathrm{O}(2)}$, if $\alpha, \beta<0[33]$.

Alternatively, one can construct the SdS algebra directly from that of Snyder by the nonunitary transformation

$x_{\mu}=\hat{x}_{\mu}+\lambda \frac{\beta}{\alpha} \hat{p}_{\mu}, \quad p_{\mu}=(1-\lambda) \hat{p}_{\mu}-\frac{\alpha}{\beta} \hat{x}_{\mu}$,

where $\hat{x}_{\mu}, \hat{p}_{\mu}$ are generators of the Snyder algebra and $\lambda$ is a free parameter [33].

\section{Phenomenological Applications}

A wide literature considers the phenomenological implications of the nonrelativistic Snyder model, especially in connection with the generalized uncertainty principle (GUP) [34,35]. However, we are interested in the relativistic case, which has obtained much less consideration. Some consequences are:

- Deformed relativistic uncertainty relations: from the deformed Heisenberg algebra, one gets

$\Delta x_{\mu} \Delta p_{\nu} \geq \frac{1}{2}\left(\eta_{\mu \nu}+\beta \Delta p_{\mu} \Delta p_{\nu}\right)$.

The spatial components essentially coincide with those considered in GUP.

ISSN 2071-0194. Ukr. J. Phys. 2019. Vol. 64, No. 11
- Modification of the perihelion shift of planetary orbits [36]: provided that the model is applicable to macroscopic phenomena, on a Schwarzschild background, the perihelion shift gets an additional contribution, $\delta \theta=\delta \theta_{\text {rel }}\left(1+\frac{5}{3} \beta m^{2}\right)$, where $m$ is the planet mass. This correction clearly breaks the equivalence principle on Planck scales.

- DSR-like effects [37,38]: no effects of time delay of cosmological photons occur, contrary to other models derived from a noncommutative geometry [39], but some higher-order effects are still present.

\section{Hopf Algebras}

In the study of noncommutative models, an important tool is given by the Hopf algebra formalism [9], especially in relation with QFT.

Since, in a noncommutative geometry, spacetime coordinates are noncommuting operators, the composition of two plane waves $e^{i p x}$ and $e^{i q x}$ gives rise to nontrivial addition rules for the momenta denoted by $p \oplus q$, that are described by the coproduct structure of a Hopf algebra $\Delta(p, q)$. The addition law is in general noncommutative.

Moreover, the opposite of the momentum is determined by the antipode of the Hopf algebra, $S(p)$, such that $p \oplus S(p)=S(p) \oplus p=0$.

The algebra associated to the Snyder model can be calculated (classically) using the geometric representation of the momentum space as a coset space mentioned above and calculating the action of the group multiplication on it [40].

Alternatively, one can use the algebraic formalism of realizations $[25,26]$ : a realization of the noncommutative coordinates $x_{\mu}$ is defined in terms of coordinates $\xi_{\mu}, p_{\mu}$ that satisfy the canonical commutation relations

$\left[\xi_{\mu}, \xi_{\nu}\right]=\left[p_{\mu}, p_{\nu}\right]=0, \quad\left[\xi_{\mu}, p_{\nu}\right]=\eta_{\mu \nu}$,

by assigning a function $x_{\mu}\left(\xi_{\mu}, p_{\mu}\right)$ that satisfies the Snyder commutation relations.

The $x_{\mu}$ and $p_{\mu}$ are now interpreted as operators acting on a function of $x_{\mu}$, as

$\xi_{\mu} \triangleright f(\xi)=\xi_{\mu} f(\xi), \quad p_{\mu} \triangleright f(\xi)=-i \partial f(\xi) / \partial \xi_{\mu}$.

In particular, it is easy to show that the most general realization of the Snyder model is given by [28]

$x_{\mu}=\xi_{\mu}+\beta \xi \cdot p p_{\mu}+\beta \chi\left(\beta p^{2}\right) p_{\mu}$, 
where the function $\chi\left(\beta p^{2}\right)$ is arbitrary and does not contribute to the commutators, but takes into account the ambiguities arising from the operator ordering of $\xi_{\mu}$ and $p_{\mu}$ into account.

In general, it can be shown that, for any noncommutative model [41],

$e^{i k x} \triangleright e^{i q \xi}=e^{i \mathcal{P}(k, q) \xi+i \mathcal{Q}(k, q)}$,

where the functions $\mathcal{P}_{\mu}$ and $\mathcal{Q}$ can be deduced from the realization. Moreover,

$e^{i k x} \triangleright 1=e^{i \mathcal{K}(k) \xi+i \mathcal{J}(k)}$

with $\mathcal{K}_{\mu}(k) \equiv \mathcal{P}_{\mu}(k, 0)$ and $\mathcal{J}(k) \equiv \mathcal{Q}(k, 0)$. The generalized addition of momenta is then given by

$k_{\mu} \oplus q_{\mu}=\mathcal{D}_{\mu}(k, q)$,

with $\mathcal{D}_{\mu}(k, q)=\mathcal{P}_{\mu}\left(\mathcal{K}^{-1}(k), q\right)$, and the coproduct is simply

$\Delta p_{\mu}=\mathcal{D}_{\mu}(p \otimes 1,1 \otimes p)$.

Note that $\mathcal{D}_{\mu}$ is independent of $\chi$. Moreover, the antipode $S\left(p_{\mu}\right)$ is $-p_{\mu}$ for all (generalized) Snyder models.

A fundamental property of the Snyder addition law is that it is nonassociative. Hence, the algebra is noncoassociative, so it is strictly not a Hopf algebra.

For the calculations, it is also useful to define a star product, that gives a representation of the product of functions of the noncommutative coordinates $x$ in terms of a deformation of the product of functions of the commuting coordinates $\xi$. In particular, from the previous results, one can calculate the star product of two plane waves:

$e^{i k \xi} \star e^{i q \xi}=e^{i \mathcal{D}(k, q) \xi+i \mathcal{G}(k, q)}$,

where

$\mathcal{G}(k, q)=\mathcal{Q}\left(\mathcal{K}^{-1}(k), q\right)-\mathcal{Q}\left(\mathcal{K}^{-1}(k), 0\right)$.

We consider now a Hermitian realization of the Snyder commutation relations

$$
\begin{aligned}
& x^{\mu}=\xi^{\mu}+\frac{\beta}{2}\left(\xi \cdot p p^{\mu}+p^{\mu} p \cdot \xi\right)= \\
& =\xi^{\mu}+\beta \xi \cdot p p^{\mu}-\frac{5 i}{2} \beta p^{\mu} .
\end{aligned}
$$

\section{4}

The request of the Hermiticity will be important for the field theory. We get

$$
\begin{aligned}
& \mathcal{D}_{\mu}(k, q)=\frac{1}{1-\beta k \cdot q}\left[\left(1+\frac{\beta k \cdot q}{1+\sqrt{1+\beta p^{2}}}\right) k_{\mu}+\right. \\
& \left.+\sqrt{1+\beta p^{2}} q_{\mu}\right] \\
& \mathcal{G}(k, q)=\frac{5 i}{2} \ln [1-\beta k \cdot q] .
\end{aligned}
$$

Hence,

$e^{i k \cdot \xi} \star e^{i q \cdot \xi}=\frac{e^{i \mathcal{D}(k, q) \cdot \xi}}{(1-\beta k \cdot q)^{5 / 2}}$.

\section{QFT in the Snyder Space}

Let us consider a QFT for a scalar field $\phi$ on the Snyder space. Usually, the field theories in noncommutative spaces are constructed by continuing to the Euclidean signature and writing the action in terms of the star product $[7,8]$.

In fact, the action functional for a free massive real scalar field $\phi(x)$ can be defined through the star product as [28]

$S_{\text {free }}[\phi]=\frac{1}{2} \int d^{4} \xi\left(\partial_{\mu} \phi \star \partial^{\mu} \phi+m^{2} \phi \star \phi\right)$.

The star product of two real scalar fields $\phi(\xi)$ and $\psi(\xi)$ can be computed by expanding them in Fourier series,

$\phi(\xi)=\int d^{4} k \tilde{\phi}(k) e^{i k \cdot \xi}$

Then, using (26),

$\int d^{4} \xi \psi(\xi) \star \phi(\xi)=\int d^{4} \xi \int d^{4} k d^{4} q \tilde{\psi}(k) \tilde{\phi}(q) \times$
$\times e^{i k \xi} \star e^{i q \xi}=\int d^{4} k d^{4} q \tilde{\psi}(k) \tilde{\phi}(q) \frac{\delta^{(4)}(\mathcal{D}(k, q))}{(1-\beta k \cdot q)^{5 / 2}}$.

But,

$\delta^{(4)}(\mathcal{D}(k, q))=\frac{\delta^{(4)}(q+k)}{\left|\operatorname{det}\left(\frac{\partial \mathcal{D}_{\mu}(k, q)}{\partial q_{\nu}}\right)\right|_{q=-k}}=$

$=\left(1+\beta k^{2}\right)^{5 / 2} \delta^{(4)}(q+k)$.

The two $\left(1+\beta k^{2}\right)^{5 / 2}$ factors cancel, and then [28]

$\int d^{4} \xi \psi(\xi) \star \phi(\xi)=\int d^{4} \xi \psi(\xi) \phi(\xi)$.

ISSN 2071-0194. Ukr. J. Phys. 2019. Vol. 64, No. 11 
This is called the cyclicity property and occurs also in other noncommutative models; it follows that the free theory is identical to the commutative one,

$S_{\text {free }}[\phi]=\frac{1}{2} \int d^{4} \xi\left(\partial_{\mu} \phi \partial_{\mu} \phi+m^{2} \phi^{2}\right)$.

The propagator is, therefore, the standard one

$G(k)=\frac{1}{k^{2}+m^{2}}$.

Note that the cyclicity property is a consequence of our choice of a Hermitian representation for the operator $x$ and can be related to the choice of a correct measure in the curved momentum space.

The interaction theory is much more difficult to be investigated. Several problems arise:

- The addition law of momenta is noncommutative and nonassociative; therefore, one must define some ordering for the lines entering a vertex and then take an average.

- The conservation law of momentum is deformed at vertices, so the loop effects may lead to the nonconservation of the momentum in a propagator.

For example, let us consider the simplest case, a $\phi^{4}$ theory $[22,23]$

$S_{\mathrm{int}}=\lambda \int d^{4} \xi \phi \star(\phi \star(\phi \star \phi))$.

The parentheses are necessary, because the star product is nonassociative. Our definition fixes this ambiguity, but other choices are possible.

With this choice, the 4-point vertex function turns out to be

$G^{(0)}\left(p_{1}, p_{2}, p_{3}, p_{4}\right)=\sum_{\sigma \in S_{4}} \delta\left(\mathcal{D}_{4}\left(\sigma\left(p_{1}, p_{2}, p_{3}, p_{4}\right)\right)\right) \times$

$\times g_{3}\left(\sigma\left(p_{1}, p_{2}, p_{3}, p_{4}\right)\right)$,

where

$\mathcal{D}_{4}\left(q_{1}, q_{2}, q_{3}, q_{4}\right)=q_{1}+\mathcal{D}\left(q_{2}, \mathcal{D}\left(q_{3}, q_{4}\right)\right)$,

$g_{3}\left(q_{1}, q_{2}, q_{3}, q_{4}\right)=e^{i \mathcal{G}\left(q_{2}, \mathcal{D}\left(q_{3}, q_{4}\right)\right)} e^{i \mathcal{G}\left(q_{3}, q_{4}\right)}$,

and $\sigma$ denotes all possible permutations of the momenta entering the vertex.

With the expressions for the propagator and the vertex, one can compute Feynman diagrams. For example, the one-loop two-point function depicted in Figure in the position space is given by

$G^{(1)}\left(\xi_{1}, \xi_{2}\right)=$

ISSN 2071-0194. Ukr. J. Phys. 2019. Vol. 64, No. 11 $=-\frac{1}{2} \frac{\lambda}{4 !} \int d^{4} p_{1} d^{4} p_{2} d^{4} \ell \frac{e^{i p_{1} \xi_{1}}}{p_{1}^{2}+m^{2}} \frac{e^{i p_{2} \xi_{2}}}{p_{2}^{2}+m^{2}} \frac{1}{\ell^{2}+m^{2}} \times$
$\times \sum_{\sigma} \delta\left(D_{4}\left(\sigma\left(p_{1}, p_{2}, \ell,-\ell\right)\right)\right) g_{3}\left(\sigma\left(p_{1}, p_{2}, \ell,-\ell\right)\right)$.

To evaluate the diagram, one must consider 24 permutations of the momenta entering the vertex. Among these, only 8 conserve the momentum (i.e. $p_{1}=-p_{2}$ ), while the remaining 16 do not.

At the linear level in $\beta$, the calculation can be done explicitly, showing stronger divergences than in the commutative theory $[22,23]$. However, the effects of momentum nonconservation cancel out.

Attempting instead a calculation at all orders in $\beta$, not all diagrams can be explicitly computed [24]. It can be shown, however, that the divergences are suppressed with respect to the noncommutative theory, and there are even indications that the integrals might be finite, at least for the interaction (34).

If instead, the renormalization is necessary, the phenomenon of UV/IR mixing could still be present, as in other noncommutative models $[7,8]$. We recall, however, that a model that avoids this problem in the Moyal theory was proposed by Grosse and Wulkenhaar [42] (GW model). Its main characteristic is that, besides the kinetic and interaction terms, its action also contains a term proportional to $\phi x^{2} \phi$. A similar mechanism can be recovered in the Snyder theory by considering a curved background (SdS model) [43].

In fact, using relation (14) between the SdS and the Snyder algebra with $\lambda=0$ and realization (24) of the Snyder algebra, the action can be reduced, at the zeroth order in $\alpha, \beta$, to

$S_{\text {free }}=\int d \xi^{4} \phi\left[p^{2}+\frac{\alpha}{\beta} \xi^{2}+m^{2}+O(\alpha, \beta)\right] \phi$,

Action (38) is identical to that of the free GW model. One may therefore hope for that, also in this case, the IR divergences are suppressed, and one can obtain a renormalizable theory.

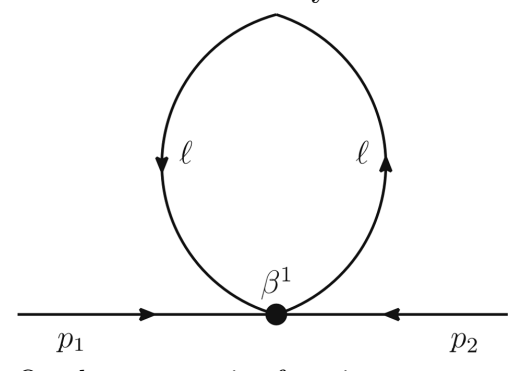

One-loop two-point function 


\section{Conclusions}

We have reviewed the present status of researches on the Snyder model, the earliest example of the noncommutative geometry, and the only one that preserves the Lorentz invariance. In particular, we concentrated on the definition of a quantum field theory defined in accord with the standard noncommutative formalism and on the issue of renormalizability. It turns out that, although an exact calculation has not been performed in full, there is good evidence of the renormalizability and the absence of UV/IR mixing, at least in the SdS model.

1. L.J. Garay. Quantum gravity and minimum length. Int. J. Mod. Phys. A 10, 145 (1995).

2. S. Hossenfelder. Minimal length scale scenarios for quantum gravity. Liv. Rev. Rel. 16, 2 (2013).

3. H.S. Snyder. Quantized space-time. Phys. Rev. 71, 38 (1947)

4. H.S. Snyder. The electromagnetic field in quantized spacetime. Phys. Rev. 72, 68 (1947).

5. A. Connes. Noncommutative Geometry (Academic Press, 1994).

6. J. Madore. An Introduction to Noncommutative Differential Geometry and Its Physical Applications (Cambridge Univ. Press, 1999).

7. M.R. Douglas, N.A. Nekrasov. Noncommutative field theory. Rev. Mod. Phys. 73, 977 (2001).

8. R.J. Szabo. Quantum field theory on noncommutative spaces. Phys. Rept. 378, 207 (2003).

9. S. Majid. Foundations of Quantum Group Theory (Cambridge Univ. Press, 1995).

10. G. Amelino-Camelia. Relativity in spacetimes with shortdistance structure governed by an observer-independent (Planckian) length scale. Int. J. Mod. Phys. D 11, 35 (2002).

11. G. Amelino-Camelia. Testable scenario for relativity with minimum length. Phys. Lett. B 510, 255 (2001).

12. J. Kowalski-Glikman. De Sitter space as an arena for doubly special relativity. Phys. Lett. B 547, 291 (2002).

13. J. Kowalski-Glikman, S. Nowak. Doubly special relativity and de Sitter space. Class. Quantum Grav. 20, 4799 (2003).

14. Y.A. Gol'fand. Quantum field theory in constant curvature p-space. Sov. Phys. JETP 16, 184 (1963).

15. Y.A. Gol'fand. On the properties of displacements in pspace of constant curvature. Sov. Phys. JETP 17, 842 (1963).

16. Y.A. Gol'fand. On the introduction of an "elementary length" in the relativistic theory of elementary particles. Sov. Phys. JETP 37, 356 (1960).

17. V.G. Kadyshevsky. On the theory of quantization of spacetime. Sov. Phys. JETP 14, 1340 (1962).
18. R.M. Mir-Kasimov. "Focusing" singularity in p-space of constant curvature. Sov. Phys. JETP 22, 629 (1966).

19. R.M. Mir-Kasimov. The Coulomb field and the nonrelativistic quantization of space. Sov. Phys. JETP 25, 348 (1967).

20. J.C. Breckenridge, T.G. Steele, V. Elias. Massless scalar field theory in a quantized space-time. Class. Quantum Grav. 12, 637 (1995).

21. T. Konopka. A field theory model with a new Lorentzinvariant energy scale. Mod. Phys. Lett. A 23, 319 (2008).

22. S. Meljanac, S. Mignemi, J. Trampetić, J. You. Nonassociative Snyder $\phi^{4}$ quantum field theory. Phys. Rev. D 96, 045021 (2017).

23. A. Franchino-Viñas, S. Mignemi. Worldline formalism in Snyder spaces. Phys. Rev. D 98, 065010 (2018).

24. S. Meljanac, S. Mignemi, J. Trampetić, J. You. UV-IR mixing in nonassociative Snyder $\phi^{4}$ theory. Phys. Rev. D 97, 055041 (2018).

25. M.V. Battisti, S. Meljanac. Modification of Heisenberg uncertainty relations in non-commutative Snyder space-time geometry. Phys. Rev. D 79, 067505 (2009).

26. M.V. Battisti, S. Meljanac. Scalar field theory on noncommutative Snyder spacetime. Phys. Rev. D 82, 024028 (2010).

27. B. Ivetić, S. Mignemi. Relative-locality geometry for the Snyder model. Int. J. Mod. Phys. D 27, 1950010 (2018).

28. S. Meljanac, D. Meljanac, S. Mignemi, R. Štrajn. Quantum field theory in generalised Snyder spaces. Phys. Lett. B 768, 321 (2017).

29. C.N. Yang. On quantized space-time. Phys. Rev. 72, 874 (1947).

30. J. Kowalski-Glikman, L. Smolin. Triply special relativity. Phys. Rev. D 70, 065020 (2004).

31. H.G. Guo, C.G. Huang, H.T. Wu. Yang's model as triply special relativity and the Snyder's model-de Sitter special relativity duality. Phys. Lett. B 663, 270-274 (2008).

32. M. Born. Reciprocity theory of elementary particles. Rev. Mod. Phys. 21, 463 (1949).

33. S. Mignemi. The Snyder-de Sitter model from six dimensions. Class. Quantum Grav. 26, 245020 (2009).

34. G. Veneziano. A stringy nature needs just two constants. Europhys. Lett. 2, 199 (1986).

35. M. Maggiore. A generalized uncertainty principle in quantum gravity. Phys. Lett. B 304, 65 (1993).

36. S. Mignemi, R. Štrajn. Snyder dynamics in a Schwarzschild spacetime. Phys. Rev. D 90, 044019 (2014).

37. S. Mignemi, A. Samsarov. Relative-locality effects in Snyder spacetime. Phys. Lett. A 381, 1655 (2017).

38. S. Mignemi, G. Rosati. Relative-locality phenomenology on Snyder spacetime. Class. Quantum Grav. 35, 145006 (2018).

39. G. Gubitosi, F. Mercati. Relative locality in $\kappa$-Poincaré. Class. Quantum Grav. 20, 145002 (2013).

ISSN 2071-0194. Ukr. J. Phys. 2019. Vol. 64, No. 11 
40. F. Girelli, E. Livine. Scalar field theory in Snyder spacetime: alternatives. JHEP 1103, 132 (2011).

41. S. Meljanac, Z. Škoda, D. Svrtan. Exponential formulas and Lie algebra type star products. SIGMA 8, 013 (2012).

42. H. Grosse, R. Wulkenhaar. Renormalisation of $\varphi^{4}$-theory on noncommutative $\mathbb{R}^{4}$ in the matrix base. Commun. Math. Phys. 256, 305 (2005).

43. A. Franchino-Viñas, S. Mignemi, in preparation.

Received 02.08.19
С. Мінъемі

МОДЕЛЬ СНАЙДЕРА ТА КВАНТОВА ТЕОРІЯ ПОЛЯ

Р ез ю м е

Дано огляд основних характерних особливостей релятивістської моделі Снайдера та її узагальнень. Обговорюється квантова теорія поля на цій основі з використанням стандартного формалізму некомутативної КТП, а також можливість отримання скінченної теорії. 